TAMKANG JOURNAL OF MATHEMATICS

Volume 33, Number 3, Autumn 2002

\title{
S-CLUSTER SETS IN FUZZY TOPOLOGICAL SPACES
}

\author{
A. A. NOUH AND M. E. EL-SHAFEI
}

\begin{abstract}
In this paper the concept of $S$-cluster fuzzy sets, of fuzzy functions and fuzzy multifunctions between fuzzy topological spaces is introduced. As an application, characterizations of fuzzy Hausdorff and $S Q_{\alpha}$-closed fuzzy topological spaces are achieved via such cluster fuzzy sets.
\end{abstract}

\section{Introduction}

The theory of cluster sets was developed long ago, and was initially aimed at the investigations of real and complex function theory. A comprehensive collection of works in this direction can be found in the classical book of Collingwood and Lohwater [4]. Weston [14] was the first to initiate the corresponding theory for functions between topological spaces basically for studyng compactness. The present paper is intended for to introduce the concept of $S$-cluster fuzzy sets of fuzzy functions and fuzzy multifunctions, which provides a new technique for studying $S Q_{\alpha}$-closedness of fuzzy topological spaces. It is shown that such cluster fuzzy sets of suitable fuzzy function can characterize fuzzy Hausdorffness. Finally, we achieve, as our prime motivation, certain characterizations of $S Q_{\alpha}$-closed space.

Let $X$ be a set of points and $I$ be the unit interval [0,1]. A fuzzy set $\mu$ in $X$ is a mapping from $X$ into $I$. The class of all fuzzy sets on $X$ denoted by $I^{X}$. For $x \in X$ and $\alpha \in(0,1]$, a fuzzy set $x_{\alpha}$ defined by

$$
x_{\alpha}(y)=\left\{\begin{array}{lll}
\alpha & : & y=x \\
0 & : & y \neq x
\end{array}\right.
$$

is called a fuzzy point in $X$. The class of all fuzzy points of $X$ denoted by $F P(X)$. Let $0_{X}$ and $1_{X}$ be, respectively, the constant fuzzy sets taking 0 and 1 on $X$. For $A \subseteq X, 1_{A}$ denotes the characteristic mapping of $A$. For every $x_{\alpha} \in F P(X)$ and $\mu \in I^{X}$, we write $X_{\alpha} \in \mu$ iff $\alpha \leq \mu(x)$. For every $\mu \in L^{X}$, denote $\operatorname{supp}(\mu)=\{x \in X: \mu(x)>0\}$, called it the support of $\mu$. For any set $A \subseteq X$, we denote the cardinality of $A$ by $|A|$. If $|A|=1$,

Received March 30, 2001; revised October 03, 2001.

2000 Mathematics Subject Classification. 54D20, 54D30, 54C60, 54C99.

$K e y$ words and phrases. Fuzzy topoloty, $Q$-neighborhood, $S$-cluster fuzzy set, $S Q_{\alpha}$-closedness, $\theta S$-closure, semi-open fuzzy sets. 
say $A=\{x\}$, then $A$ is called degenerate. A fuzzy set $\mu$ is called finite (resp. degenerate) if $|\operatorname{supp}(\mu)|$ is finite (resp. $\mid \operatorname{supp}(\mu)=1$ ). A fuzzy set $\mu$ is called quasi-coincident with a fuzzy set $\rho$,denoted by $\mu q \rho$ [12], iff there exists $x \in X$ such that $\mu(x)+\rho(x)>1$. If $\mu$ is not quasi-coincident with $\rho$, then we write $\mu \bar{q} \rho$.

In what follows, we use the concept of a fuzzy topological space (fts, for short) as introduced by Chang [3]. A fuzzy set $\mu \in I^{X}$ is called semi-open [9] if for some open fuzzy set $\eta, \eta \leq \mu \leq c \ell(\eta)$, where $c \ell(\eta)$ denotes the fuzzy closure of $\eta$ in $X$. The complements of semi-open fuzzy sets are called semi-closed. Let $x_{\alpha} \in F P(X)$ and $\mu \in I^{X}$, by $N_{\mu}, N_{x_{\alpha}}^{Q}, S O N_{x_{\alpha}}^{Q}$ and $1_{X} \backslash \mu=\mu^{\prime}$, we mean, the open neighbourhood system of $\mu$, open $Q$-neighbourhood ( $Q$-nbd, for short) system of $x_{\alpha}$, the semi-open $Q$-neighbourhood (S.Q-nbd, for short) system of $x_{\alpha}$ and the pseudo-complement of $\mu$. For any fuzzy set $\mu \in I^{X}$, the $\theta$-closure [9] ( $\theta$-semiclosure [9]) of $\mu$, denoted by $\theta . c \ell(\mu)$ (resp. $\theta S . c \ell(\mu)$ ), is defined by $x_{\alpha} \in \theta . c l(\mu)$ (resp. $x_{\alpha} \in \theta S . c l(\mu)$ ) iff for every $\eta \in N_{x_{\alpha}}^{Q}$ (resp. $\left.\eta \in S O N_{x_{\alpha}}^{Q}\right), c \ell(\eta) q \mu$. The fuzzy set $\mu$ is called $\theta$-closed [9] $(\theta$-semiclosed [9]) if $\mu=\theta . c \ell(\mu)$ (resp. $\mu=\theta S . c \ell(\mu)$ ). It is known [9] that $\theta . c \ell(\mu)$ need not be $\theta$-closed, but it is so if $\mu$ is open.

Theorem 1.1.([12]) Let $\left\{\mu_{j}: j \in J\right\} \subseteq I^{X}$ and $x_{\alpha} \in F P(X)$. Then:

(i) $x_{\alpha} q \bigvee_{j \in J} \mu_{j}$ iff $\left(\exists j_{0} \in J\right)\left(x_{\alpha} q \mu_{j 0}\right)$.

(ii) If $x_{\alpha} q \bigwedge_{j \in J} \mu_{j}$, then $\left(\forall_{j} \in J\right)\left(x_{\alpha} q \mu_{j}\right)$. The converse is true if $J$ is finite.

Definition 1.2.([2]) A fuzzy grill on $X$ is a nonempty subset $\Omega \subseteq I^{X}$ such that:

(i) $\mu \in \Omega$ and $\eta \leq \mu$ implies $\eta \in \Omega$.

(ii) $\mu \vee \eta \in \Omega$ implies $\mu \in \Omega$ or $\eta \in \Omega$.

Definition 1.3.([11]) A fuzzy filterbase on $X$ is a nonempty subset $\beta \subseteq I^{X}$ such that:

(i) $0_{X} \notin \beta$.

(ii) If $\mu_{1}, \mu_{2} \in \beta$, then $\exists \mu_{3} \in \beta$ such that $\mu_{3} \leq \mu_{1} \wedge \mu_{2}$.

The fuzzy filter $\mathcal{F}$ generated by $\beta$ is difined by $\mathcal{F}=\left\{\mu \in I^{X}: \eta \leq \mu\right.$ for some $\left.\eta \in \beta\right\}$. A fuzzy filterbase $\mathcal{F}$ on a fts $(X, \tau)$ is said to $\theta S$-adhere at a fuzzy point $x_{\alpha} \in F P(X)$, denoted as $x_{\alpha} \in \theta S . a d h(\mathcal{F})$ if $\left(\forall \eta \in S O N_{x \alpha}^{Q}\right)(\forall \lambda \in \mathcal{F})(c \ell(\eta) q \lambda)$. A fuzzy grill $\Omega$ on $X$ is said to $\theta S$-converge to a fuzzy point $x_{\alpha} \in F P(X)$, if for each $\mu \in S O N_{x_{\alpha}}^{Q}$, there corresponds some $\lambda \in \Omega$ with $\lambda \leq c(\mu)$.

Each mapping $f: I^{X} \rightarrow I^{Y}$ considered in this paper is induced from a crisp mapping $f: X \rightarrow Y$ as usual, i.e. for $\mu \in I^{X}, \eta \in I^{Y}, x \in X$ and $y \in Y$, we define $f(\mu)(y)=$ $\bigvee\{\mu(x): x \in X, f(x)=y\}$ and $f^{-1}(\eta)(x)=\eta(f(x))$.

\section{S-cluster Fuzzy Set of Fuzzy Functions}

Definition 2.1. Let $f: I^{X} \rightarrow I^{Y}$ be a function and $x_{\alpha} \in F P(X)$. The $S$-cluster fuzzy set of $f$ at $x_{\alpha}$, denoted by $S\left(f, x_{\alpha}\right)$ is given by $\wedge\left\{\theta . c \ell(f(c \ell(\mu))): \mu \in S O N_{x_{\alpha}}^{Q}\right\}$. 
In the next theorem, we characterize the $S$-cluster fuzzy set of a function at some fuzzy point between fuzzy topological spaces.

Theorem 2.2. For any function $f: I^{X} \rightarrow I^{Y}$ and $y_{v} \in F P(Y)$, the following statements are equivalent:

(i) $y_{v} \in S\left(f, x_{\alpha}\right)$.

(ii) The fuzzy filterbase $f^{-1}\left(c \ell\left(N_{y_{v}}^{Q}\right)\right) \quad \theta S$-adheres at $x_{\alpha}$.

(iii) There is a fuzzy grill $\Omega$ on $X$ such that $\Omega \theta S$-converges to $x_{\alpha}$ and $y_{v} \in \wedge\{\theta . c \ell(f(\mu))$ $: \mu \in \Omega\}$.

Proof. (i) $\Longrightarrow$ (ii): Let $y_{v} \in S\left(f, x_{\alpha}\right)$. Then for each $\mu \in S O N_{x_{\alpha}}^{Q}$ and each $\eta \in$ $N_{y_{v}}^{Q}, c \ell(\eta) q f(c \ell(\mu))$. So, for each $\mu \in S O N_{x_{\alpha}}^{Q}$ and each $\eta \in N_{y_{v}}^{Q}, f^{-1}(c \ell(\eta)) q \mu$ and so $f^{-1}(c \ell(\eta)) \wedge \mu \neq \emptyset$. It is easy to verify that the family $\left\{f^{-1}(c \ell(\eta)): \eta \in N_{y_{v}}^{Q}\right\}$ is a fuzzy filterbase on $X \quad \theta S$-adheres at $x_{\alpha}$.

(ii) $\Longrightarrow$ (iii): Let $\mathcal{F}$ be the fuzzy filter on $X$ generated by the fuzzy filterbase $f^{-1}\left(c\left(N_{y_{v}}^{Q}\right)\right)$. Then $\Omega=\left\{\mu \in I^{X}: \mu q \lambda\right.$ for each $\left.\lambda \in \mathcal{F}\right\}$ is a fuzzy grill on $X$. By (ii), for each $\rho \in S O N_{x_{\alpha}}^{Q}$ and each $\eta \in N_{y_{v}}^{Q}, c \ell(\rho) q f^{-1}(c \ell(\eta))$. Hence $\lambda q c \ell(\rho)$ for each $\lambda \in \mathcal{F}$ and each $\rho \in S O N_{x_{\alpha}}^{Q}$. Consequently, $c \ell(\rho) \in \Omega$ for all $\rho \in S O N_{x_{\alpha}}^{Q}$, which proves that $\Omega \theta S$-converges to $x_{\alpha}$. Now, the definition of $\Omega$ yields that $f(\mu) q c \ell(\eta)$ for all $\eta \in N_{y_{v}}^{Q}$ and all $\mu \in \Omega$. Then $y_{v} \in \theta$.cl $(f(\mu))$ for all $\mu \in \Omega$. Hence $y_{v} \in \wedge\{\theta . c \ell(f(\mu)): \mu \in \Omega\}$.

(iii) $\Longrightarrow$ (i): Let $\Omega$ be a fuzzy grill on $X$ such that $\Omega \quad \theta S$-converges to $x_{\alpha}$, and $y_{v} \in \wedge\{\theta . c \ell(f(\mu)): \mu \in \Omega\}$. Then $\left\{c \ell(\rho): \rho \in S O N_{x_{\alpha}}^{Q}\right\} \subseteq \Omega$ and $y_{v} \in \theta . c \ell(f(\lambda))$ for each $\lambda \in \Omega$. Hence, in particular, $y_{v} \in \operatorname{\theta } . c \ell(f(c \ell(\rho)))$ for all $\rho \in S O N_{x_{\alpha}}^{Q}$. So $y_{v} \in \wedge\left\{\theta . c \ell(f(c \ell(\rho))): \rho \in S O N_{x_{\alpha}}^{Q}\right\}=S\left(f, x_{\alpha}\right)$.

Definition 2.3. A fts $(X, \tau)$ is called fuzzy Hausdorff space $\left(F T_{2}\right.$, for short $)$ iff $\left(\forall x_{\alpha}, y_{v} \in F P(X), x \neq y\right)\left(\exists \mu \in N_{x_{\alpha}}^{Q}\right)\left(\exists \eta \in N_{y_{v}}^{Q}\right)(\mu \bar{q} \eta)$.

In what follows, we show that $S$-cluster fuzzy sets of a function at some fuzzy point between fuzzy topological spaces may be used to assertain the fuzzy Hausdorffness of the codomain space.

Theorem 2.4. Let $f: I^{X} \rightarrow I^{Y}$ be a function on a fts $(X, \tau)$ onto a fts $(Y, \Delta)$. Then $(Y, \Delta)$ is $F T_{2}$ if $S\left(f, x_{\alpha}\right)$ is degenerate for each $x_{\alpha} \in F P(X)$.

Proof. Let $y_{\alpha}^{1}, y_{v}^{2} \in F P(X)$ such that $y^{1} \neq y^{2}$. As $f$ is a surjection, there are $x_{\alpha}^{1}$, $x_{v}^{2} \in F P(X)$ such that $x^{1} \neq x^{2}$ and $f\left(x^{i}\right)=y^{i}$ for $i=1,2$. Now, since $S\left(f, x_{\alpha}\right)$ is degenerate for each $x_{\alpha} \in F P(X), y_{v}^{2}=f\left(x_{v}^{2}\right) \notin S\left(f, x_{\alpha}^{1}\right)$. Thus, there are $\eta \in N_{y_{v}^{2}}^{Q}$ and $\mu \in S O N_{x_{\alpha}^{1}}^{Q}$ such that $c(\eta) \bar{q} f(c \ell(\mu))$ and so $f(c \ell(\mu)) \leq 1_{Y} \backslash c \ell(\eta)$. Then $\eta \in$ $N_{y_{\alpha}^{2}}^{Q}, 1_{Y} \backslash c \ell(\eta) \in N_{y_{v}^{1}}^{Q}$ and $\eta \bar{q}\left(1_{Y} \backslash c \ell(\eta)\right)$ which proves that $(Y, \Delta)$ is $F T_{2}$.

Definition 2.5.([7]) A function $f: I^{X} \rightarrow I^{Y}$ is called a fuzzy $\theta S$-irresolute iff for each $x_{\alpha} \in F P(X)$ and each $\eta \in S O N_{f\left(x_{\alpha}\right)}^{Q}$, there is $\mu \in S O N_{x_{\alpha}}^{Q}$ such that $f(c \ell(\mu)) \leq \eta$. 
Theorem 2.6. Let $f: I^{X} \rightarrow I^{Y}$ be a fuzzy $\theta S$-irresolute function with $(Y, \Delta)$ a $F T_{2}$-space. Then $S\left(f, x_{\alpha}\right)$ is degenerate for each $x_{\alpha} \in F P(X)$.

Proof. Let $x_{\alpha} \in F P(X)$. As $f$ is fuzzy $\theta S$-irresolute, for any $\eta \in S O N_{f\left(x_{\alpha}\right)}^{Q}$, there is $\mu \in S O N_{x_{\alpha}}^{Q}$ such that $f(c \ell(\mu)) \leq \eta$. Then $S\left(f, x_{\alpha}\right)=\wedge\left\{\theta . c \ell(f(c \ell(\mu))): \mu \in S O N_{x_{\alpha}}^{Q}\right\} \subseteq$ $\wedge\left\{\theta . c \ell(\eta): \eta \in S O N_{f\left(x_{\alpha}\right)}^{Q}\right\}$. Let $y_{\alpha} \in F P(Y)$ with $y \neq f(x)$. As $(Y, \Delta)$ is $F T_{2}$, then there are $\rho_{1} \in N_{y_{\alpha}}^{Q}$ and $\rho_{2} \in N_{f\left(x_{\alpha}\right)}^{Q}$ such that $\rho_{1} \bar{q} \rho_{2}$. Obviously, as $\rho_{1} \bar{q} c \ell\left(\rho_{2}\right), y_{\alpha} \notin$ $c \ell\left(\rho_{2}\right)=\theta . c \ell\left(\rho_{2}\right)$. As $\rho_{2} \in N_{f\left(x_{\alpha}\right)}^{Q} \subseteq S O N_{f\left(x_{\alpha}\right)}^{Q}, y_{\alpha} \notin \wedge\left\{\theta . c \ell(\eta): \eta \in S O N_{f\left(x_{\alpha}\right)}^{Q}\right\}$ and hence $y_{\alpha} \notin S\left(f, x_{\alpha}\right)$. Thus $S\left(f, x_{\alpha}\right)=\left\{f\left(x_{\alpha}\right)\right\}$.

Combining the lase two results, we get the following characterization for the fuzzy Hausdorffness of the codomain space of a kind of function in terms of the degeneracy of its $S$-cluster fuzzy set.

Theorem 2.7. $f: I^{X} \rightarrow I^{Y}$ be a fuzzy $\theta S$-irresolute function on a fts $(X, \tau)$ onto a fts $(Y, \Delta)$. Then $(Y, \Delta)$ is $F T_{2}$ iff $S\left(f, x_{\alpha}\right)$ is degenerate for each $x_{\alpha} \in F P(X)$.

We have just seen that degeneracy of the $S$-cluster fuzzy set of an arbitrary fuzzy function is a sufficient condition for the fuzzy Hausdorffness of the codomain space. We thus like to examine some other situations when the $S$-cluster fuzzy sets are degenerate, thereby ensuring the fuzzy Hausdorffness of the codomain space of the fuzzy function concerned. To this end, we recall the following definition.

Definition 2.8.([9]) A fts $(X, \tau)$ is called fuzzy almost regular $\left(F A R_{2}\right.$, for short) iff $\left(\forall x_{\alpha} \in F P(X)\right)(\forall \lambda \in R C F(X, \tau))\left(x_{\varepsilon} \notin \lambda\right)\left(\exists \eta \in N_{x_{\alpha}}^{Q}\right)\left(\exists \rho \in N_{\lambda}\right)(\eta \bar{q} \rho)$, where $R C F(X, \tau)$ denotes the class of all regular closed fuzzy sets in $(X, \tau)$.

Theorem 2.9.([9]) In any $F A R_{2}$-space $(X, \tau), \theta . c \ell(\mu)$ is $\theta$-closed fuzzy set for each $\mu \in I^{X}$.

Definition 2.10.([7]) A funciton $f: I^{X} \rightarrow I^{Y}$ is called fuzzy $\theta$-closed if the image of each $\theta$-closed fuzzy set of a fts $(X, \tau)$ is a $\theta$-closed fuzzy set of a $\mathrm{fts}(Y, \Delta)$.

Theorem 2.11. Let $f: I^{X} \rightarrow I^{Y}$ be a fuzzy $\theta$-closed function from a $F A R_{2}$-space $(X, \tau)$ into a fts $(Y, \Delta)$. If $f^{-1}\left(y_{v}\right)$ is $\theta$-closed in $(X, \tau)$ for all $y_{v} \in F P(Y)$, then $S\left(f, x_{\alpha}\right)$ is degenerate for each $x_{\alpha} \in F P(X)$.

Proof. Since, $c(\mu) \leq \theta . c \ell(\mu)$ for each $\mu \in I^{X}$, then $S\left(f, x_{\alpha}\right)=\wedge\{\theta . c \ell(f(c \ell(\mu)))$ : $\left.\mu \in S O N_{x_{\alpha}}^{Q}\right\} \leq \wedge\left\{\theta . c \ell(f(\theta . c \ell(\mu))): \mu \in S O N_{x_{\alpha}}^{Q}\right\}$. As $(X, \tau)$ is $F A R_{2}, \theta \cdot c \ell(\mu)$ is $\theta$-closed for all $\mu \in S O N_{x_{\alpha}}^{Q}$. Since $f$ is a fuzzy $\theta$-closed function, $\theta . c \ell(f(\theta . c \ell(\mu)))=f(\theta . c \ell(\mu))$ for each $\mu \in S O N_{x_{\alpha}}^{Q}$. Thus $S\left(f, x_{\alpha}\right) \leq \wedge\left\{f(\theta . c \ell(\mu)): \mu \in S O N_{x_{\alpha}}^{Q}\right\}$. Now, let $y_{v} \in F P(Y)$ such that $y \neq f(x)$. Then since $f^{-1}\left(y_{v}\right)$ is $\theta$-closed and $x_{\alpha} \notin f^{-1}\left(y_{v}\right)$, there is some $\rho \in N_{x_{\alpha}}^{Q}$ such that $c \ell(\rho) \bar{q} f^{-1}\left(y_{v}\right)$. So, $y_{v} \notin f(c \ell(\rho))=f(\theta . c \ell(\rho))$ (as $\rho$ is an open fuzzy set) and hence $y_{v} \notin \wedge\left\{f(\theta . c \ell(\mu)): \mu \in S O N_{x_{\alpha}}^{Q}\right\}$. Thus, we conclude that $y_{v} \notin S\left(f, x_{\alpha}\right)$, which proves that $S\left(f, x_{\alpha}\right)$ is degenerate. 
Theorem 2.12. Let $f: I^{X} \rightarrow I^{Y}$ be a fuzzy $\theta$-closed injection funciton, where $(X, \tau)$ is a $F A R_{2}$ and $F T_{2}$-space. Then $S\left(f, x_{\alpha}\right)$ is degenerate for each $x_{\alpha} \in F P(X)$.

Proof. Since the fts $(X, \tau)$ is $F A R_{2}$ and the mapping $f$ is a fuzzy $\theta$-closed, we have $\theta . c \ell(f(\theta . c \ell(\mu)))=f(\theta . c \ell(\mu))$ for any $\mu \in S O N_{x_{\alpha}}^{Q}$ and, hence

$$
\begin{aligned}
S\left(f, x_{\alpha}\right) & =\wedge\left\{\theta \cdot c \ell(f(c \ell(\mu))): \mu \in S O N_{x_{\alpha}}^{Q}\right\} \\
& \leq \wedge\left\{\theta \cdot c \ell(f(\theta \cdot c \ell(\mu))): \mu \in S O N_{x_{\alpha}}^{Q}\right\} \\
& =\wedge\left\{f(\theta \cdot c \ell(\mu)): \mu \in S O N_{x_{\alpha}}^{Q}\right\} .
\end{aligned}
$$

For each $x_{\alpha}^{1} \in F P(X)$ with $x \neq x^{1}, f\left(x_{\alpha}\right) \neq f\left(x_{\alpha}^{1}\right)$ as $f$ is injective. By the fuzzy Hausdorffness of $(X, \tau)$, there are $\mu \in N_{x_{\alpha}}^{Q}$ and $\eta \in N_{x_{\alpha}^{1}}^{Q}$ such that $\mu \bar{q} \eta$. Obviously, $\mu \bar{q} c \ell(\eta)$. So $x_{\alpha}^{1} \notin \theta . c \ell(\mu)$ and hence $f\left(x_{\alpha}^{1}\right) \notin f(\theta . c \ell(\mu))$. Since $N_{x_{\alpha}}^{Q} \subseteq S O N_{x_{\alpha}}^{Q}$, then $\mu \in S O N_{x_{\alpha}}^{Q}$ and hence by equation $(*)$, we have $f\left(x_{\alpha}^{1}\right) \notin S\left(f, x_{\alpha}\right)$. Thus, $S\left(f, x_{\alpha}\right)$ is degenerate for each $x_{\alpha} \in F P(X)$.

Definition 2.13.([9]) A fts $(X, \tau)$ is called fuzzy regular $\left(F R_{2}\right.$, for short) iff ( $\forall x_{\alpha} \in$ $F P(X))\left(\forall \lambda \in \tau^{\prime}\right)\left(x_{\alpha} \notin \lambda\right)\left(\exists \eta \in N_{x_{\alpha}}^{Q}\right)\left(\exists \rho \in N_{\lambda}\right)(\eta \bar{q} \rho)$, where $\tau^{\prime}$ represents the class of all closed fuzzy sets in $(X, \tau)$.

Now, Theorem 2.12., is equivalent to the following apparently weaker result when $(X, \tau)$ is $F R_{2}$.

Theorem 2.14. If $f: I^{X} \rightarrow I^{Y}$ is a fuzzy $\theta$-closed injection, where $(X, \tau)$ is a $F R_{2}$-space, then $S\left(f, x_{\alpha}\right)$ is degenerate for each $x_{\alpha} \in F P(X)$.

Proof. It is known that in a $F R_{2}$-space $(X, \tau), \theta \cdot c \ell(\mu)=c \ell(\mu)$ for any $\mu \in I^{X}$. Since $(X, \tau)$ is $F T_{3}$ and $f$ is a fuzzy $\theta$-colsed injection, $\left\{f\left(x_{\alpha}\right)\right\} \leq S\left(f, x_{\alpha}\right)=\wedge\{f(c \ell(\mu)): \mu \in$ $\left.S O N_{x_{\alpha}}^{Q}\right\} \leq \wedge\left\{f(c \ell(\mu)): \mu \in N_{x_{\alpha}}^{Q}\right\}=\left\{f\left(x_{\alpha}\right)\right\}$. Thus $S\left(f, x_{\alpha}\right)=\left\{f\left(x_{\alpha}\right)\right\}$.

Note that the above result is indeed equivalent to that of Theorem 2.9 follows from the following considerations: For any fuzzy set $\mu \in I^{X}$ in a fts $(X, \tau), \theta$-closure of $\mu$ in $(X, \tau)$ is the same as that in $\left(X, \tau_{S}\right)$, where $\left(X, \tau_{S}\right)$ denotes the fuzzy semiregularization space [9] of $(X, \tau)$. Moreover, it is known [9] that $(X, \tau)$ is $F T_{2}\left(F A R_{2}\right)$ iff $\left(X, \tau_{S}\right)$ is $F T_{2}\left(F R_{2}\right)$. Now, since $S O\left(X, \tau_{S}\right) \leq S O(X, \tau)$, it follows that $S\left(f, x_{\alpha}\right)=S\left(f:(X, \tau) \rightarrow Y, x_{\alpha}\right) \leq$ $S\left(f:\left(X, \tau_{S}\right) \rightarrow Y, x_{\alpha}\right)$. So, $S\left(f, x_{\alpha}\right)$ is degenerate for each $x_{\alpha} \in F P(X)$ if $(X, \tau)$ is an $F A R_{2}$ and $F T_{2}$ space and $f: I^{X} \rightarrow I^{Y}$ is a nonempty fuzzy $\theta$-closed injection.

\section{3. $S$-cluster Sets of Fuzzy Multifunctions and $S Q_{\alpha}$-closedness}

Definition 3.1.([10]) Let $(X, T)$ be a topological space in the classical sense and $(Y, \Delta)$ be a fts. A map $F: X \rightarrow I^{Y}$ is called a fuzzy multifunction iff for each $x \in X, F(x)$ is a nonempty fuzzy set in $Y$.

In the following, unless otherewise is stated, by $F: X \rightarrow I^{Y}$ we will mean that $F$ is a fuzzy multifunction from a classical topological space $(X, T)$ to a $\mathrm{fts}(Y, \Delta)$. Let 
$(X, T)$ be a classical topological space, $x \in X$ and $A \subseteq X$, by $N_{x}$ (resp. $N_{A}$ ) and $S O N_{x}$ (resp. $S O N_{A}$ ), we mean, the open neighbourhood system of $x$ (resp. of $A$ ) and the semi-open neighbourhood system of $x$ (resp. of $A$ ).

Definition 3.2.([10]) For a fuzzy multifunction $F: X \rightarrow I^{Y}$, the lower inverse $F^{-}(\eta)$ of a fuzzy set $\eta$ in $Y$ is defined as: $F^{-}(\eta)=\{x \in X: F(x) q \eta\}$.

Definition 3.3. Let $F: X \rightarrow I^{Y}$ be a fuzzy multifunction and $x \in X$. Then the $S$ cluster fuzzy set of $F$ at $x$, denoted by $S(F, x)$ is defined to be the set $\wedge\{\theta \cdot c \ell(F(c \ell(U)))$ : $\left.U \in S O N_{x}\right\}$.

Definition 3.4.([8]) Let $(X, \tau)$ be a fts, $\alpha \in(0,1]$ and $\mu \in I^{X}$. Then

(i) The familly $\mathcal{U}=\left\{\eta_{j}: j \in J\right\} \subseteq S O(X, \tau)$ is called a semi-open $Q_{\alpha}$-cover of $\mu$ iff $(\forall x \in X$ with $\mu(x) \geq \alpha)(\exists j \in J)\left(x_{\alpha} q \eta_{j}\right)$.

(ii) A subfamilly $\mathcal{U}_{0}$ of an $Q_{\alpha}$-cover $\mathcal{U}$ of $\mu$, which is also a $Q_{\alpha}$-cover of $\mu$, is called an $Q_{\alpha}$-subcover of $\mu$.

(iii) A fuzzy set $\mu$ is called $S Q_{\alpha}$-closed if each semi-open $Q_{\alpha}$-cover $\mathcal{U}$ of $\mu$ there exists a finite subfamily $\mathcal{U}_{0}$ of $\mathcal{U}$ such that $\left\{c \ell(\eta): \eta \in \mathcal{U}_{0}\right\}$ is an $Q_{\alpha}$-cover of $\mu$.

(iv) A fts $(X, \tau)$ is called $S Q_{\alpha}$-closed iff $1_{X}$ is $S Q_{\alpha}$-closed.

We now turn our attention to the characterizations of $S Q_{\alpha}$-closedness via $S$-cluster fuzzy sets. We need the following two lemmas for this purpose.

Lemma 3.5. A fuzzy set $\mu$ in a fts $(X, \tau)$ is an $S Q_{\alpha}$-closed iff for every fuzzy filterbase $\mathcal{F}$ on $X$ such that $\lambda \wedge \eta(x) \geq \alpha$ for all $\lambda \in \mathcal{F}$ and for all $\eta \in S O N_{\mu}^{Q_{\alpha}}$, then $\mu \wedge \theta \operatorname{S.adh}(\mathcal{F})(x) \geq \alpha$ for some $x \in X$.

Proof. Let $\mu \in I^{X}$ be an $S Q_{\alpha}$-closed and $\mathcal{F}$ be a fuzzy filterbase on $X$ and assume that $\mu \wedge \theta \operatorname{S.adh}(\mathcal{F})(x)<\alpha$ for each $x \in X$. Then, for all $x_{\alpha} \in \mu$, we have $x_{\alpha} \notin \theta \operatorname{S.adh}(\mathcal{F})$ and so $\left(\exists \eta_{x_{\alpha}} \in S O N_{x_{\alpha}}^{Q}\right)\left(\exists \lambda_{x_{\alpha}} \in \mathcal{F}\right)\left(c \ell\left(\eta_{x_{\alpha}}\right) \bar{q} \lambda_{x_{\alpha}}\right)$. The family $\mathcal{U}=\left\{\eta_{x_{\alpha}}: x_{\alpha} \in \mu\right\}$ is a semi-open $Q_{\alpha}$-cover of $\mu$. By the $S Q_{\alpha}$-closedness of $\mu$, there exists a finite subset $\mu^{*}$ of $\mu$ such that the family $\mathcal{U}_{0}=\left\{c \ell\left(\eta_{x_{\alpha}}\right): X_{\alpha} \in \mu^{*}\right\}$ is an $Q_{\alpha}$-cover of $\mu$. Choose $\lambda \in \mathcal{F}$ with $\lambda \leq \wedge\left\{\lambda_{x_{\alpha}} \in \mathcal{F}: x_{\alpha} \in \mu^{*}\right\}$. Put $\eta=\bigvee\left\{\eta_{x_{\alpha}} \in S O N_{x_{\alpha}}^{Q}: x_{\alpha} \in \mu^{*}\right\}$. Then $\eta \in S O N_{x_{\alpha}}^{Q}$ and $c \ell(\eta) \bar{q} \lambda$. Since $x_{\alpha} \in \mu, x_{\alpha} q \eta$ and $c \ell(\eta) \bar{q} \lambda$, then $\lambda(x) \leq 1_{X} \backslash c \ell(\eta)(x)<\alpha \leq \mu(x)$ for each $x \in X$. Hence $(\mu \wedge \lambda)(x)<\alpha$ for each $x \in X$, a contradiction. Conversely, suppose that $\mu$ is not $S Q_{\alpha}$-closed. Then there exists a semi-open $Q_{\alpha}$-cover $\mathcal{U}=\left\{\eta_{j}: j \in J\right\}$ of $\mu$ such that for every finite subset $J_{0}$ of $J$, the family $\mathcal{U}_{0}=\left\{c \ell\left(\eta_{j}\right): j \in J_{0}\right\}$ is not $Q_{\alpha}$-cover of $\mu$. Then, there exists $x_{\alpha} \in \mu$ such that for all $\left.c \ell\left(\eta_{j}\right) \in \mathcal{U}_{0}\right\}$ we have $x_{\alpha} \bar{q} c \ell\left(\eta_{j}\right)$ and so $x_{\alpha} \bar{q} \bigvee_{j \in J_{0}} c \ell\left(\eta_{j}\right)$. Hence $x_{\alpha} \in \bigwedge_{j \in J_{0}}\left(1_{X} \backslash c \ell\left(\eta_{j}\right)\right)$ and so $\bigwedge_{j \in J_{0}}\left(\left(1_{X} \backslash c \ell\left(\eta_{j}\right)\right) \wedge \mu\right)(x) \geq \alpha$ for some $x \in X$. So, $\mathcal{F}=\left\{\mu \wedge\left(1_{X} \backslash c \ell\left(\eta_{j}\right)\right): j \in J_{0}\right\}$ is a fuzzy filterbase on $\mu$. By hypothesis, we have $x_{\alpha} \in \mu \wedge \theta S . a d h(\mathcal{F})$. Assume, $\eta_{j} \in S O N_{x_{\alpha}}^{Q}$ and let $J_{0}=\{j\}$. Since, $x_{\alpha} \in \theta \operatorname{S.adh}(\mathcal{F})$, then $c \ell\left(\eta_{j}\right) q\left(\mu \wedge\left(1_{X} \backslash \eta_{j}\right)\right)$ and so $\left.c \ell\left(\eta_{j}\right) q\left(1_{X}\right) \backslash c \ell\left(\eta_{j}\right)\right)$ which is impossible. 
Lemma 3.6.([8]) For any fts $(X, \tau)$ and $\alpha \in(0,1]$ we have:

(i) A fts $(X, \tau)$ is $S Q_{\alpha}$-closed iff every fuzzy filterbase $\theta S$-adheres in $X$.

(ii) Any $\theta$-semiclosed fuzzy set of an $S Q_{\alpha}$-closed space is $S Q_{\alpha}$-closed.

Definition 3.7. For a fuzzy multifunction $F: X \rightarrow I^{Y}$ and a subset $A$ of $X$, the notation $S(F, A)$ stands for the set $\vee\{S(F, x): x \in A\}$.

Theorem 3.8. For any topological space $(X, T)$ and $\alpha \in(0,1]$, the following statements are equivalent:

(i) $1_{X}$ is $S Q_{\alpha}$-closed.

(ii) $S(F, A) \supseteq \wedge\left\{\theta \cdot c \ell(F(U)): U \in S O N_{A}\right\}$ for each $\theta$-semiclosed subset $A$ of $X$, for each fts $(Y, \Delta)$ and each fuzzy multifunction $F: X \rightarrow I^{Y}$.

(iii) $S(F, A) \supseteq \wedge\left\{\theta S . c \ell(F(U)): U \in S O N_{A}\right\}$ for each $\theta$-semiclosed subset $A$ of $X$, for each fts $(Y, \Delta)$ and each fuzzy multifunction $F: X \rightarrow I^{Y}$.

Proof. (i) $\Longrightarrow$ (ii): Let A be any $\theta$-semiclosed subset of $X$. Since, $1_{X}$ is $S Q_{\alpha}$-closed, then by Lemma 3.6(ii), $1_{A}$ is $S Q_{\alpha}$-closed. Now, let $z_{\alpha} \in \wedge\left\{\theta . c \ell(F(W)): W \in S O N_{A}\right\}$. Then for all $\eta \in N_{z_{\alpha}}^{Q}$ and for each $U \in S O N_{A}, c \ell(\eta) q F(U)$ and so $F^{-}(c \ell(\eta)) \cap U \neq \emptyset$. Thus $\mathcal{F}=\left\{F^{-}(c \ell(\eta)): \eta \in N_{z_{\alpha}}^{Q}\right\}$ is clearly a fuzzy filterbase on $X$, satisfying the condition of Lemma 3.5. Hence $\left(1_{A} \wedge \theta S . a d h(\mathcal{F})\right)(x) \geq \alpha$. Then $x \in A$, and for all $U \in S O N_{x}$ and each $\eta \in N_{z_{\alpha}}^{Q}, c \ell\left(1_{U}\right) q F^{-}(c \ell(\eta))$, i.e., $F(c \ell(U)) q c \ell(\eta)$ and so $z_{\alpha} \in$ $S(F, x) \subseteq S(F, A)$.

(ii) $\Longrightarrow$ (iii): Obvious.

(iii) $\Longrightarrow$ (i): In order to show that $1_{X}$ is $S Q_{\alpha}$-closed, it is enough to prove, by virtue of Lemma 3.6(i), that every fuzzy filterbase $\mathcal{F}$ on $X \theta S$-adheres at some $x_{\alpha} \in F P(X)$. Let $\mathcal{F}$ be a fuzzy filterbase on $X$. Take $y^{0} \notin X$, and construct $Y=X \cup\left\{y^{0}\right\}$. Define $\Delta=\left\{\eta \in I^{Y}: y_{\alpha}^{0} \bar{q} \eta\right\} \cup\left\{\eta \in I^{Y}: y_{\alpha}^{0} q \eta, \lambda \leq \eta\right.$ for some $\left.\lambda \in \mathcal{F}\right\}$. By Theorem 1.1, it is easy to verify that $\Delta$ is a fuzzy topoloty on $Y$. Consider the function $\Psi: X \rightarrow Y$ by $\Psi(x)=x$. In order to avoid possible confusion, let us denote the closure and $\theta S$-closure of a fuzzy

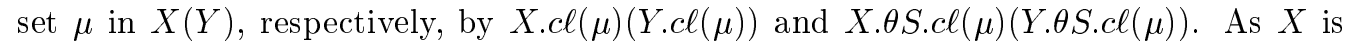
$\theta$-semiclosed in $X$, by the given codition, $S(\Psi, x) \supseteq \wedge\left\{Y \cdot \theta S . c \ell(\Psi(U)): U \in S O N_{X}\right\}=$ $\wedge\left\{Y . \theta S . c \ell(U): U \in S O N_{X}\right\}=Y . \theta S . c \ell(X)$. We consider $y_{\alpha}^{0} \in F P(Y)$ and $\rho_{0} \in S O N_{y_{\alpha}}^{Q}$. There is some $\eta \in \Delta$ such that $\eta \leq \rho_{0} \leq Y . c \ell(\eta)$. If $y_{\alpha}^{0} \bar{q} \eta$, then $\eta \leq 1_{X}$ and hence $Y . c \ell(\eta) q 1_{X}$. If on the other hand, $y_{\alpha}^{0} q \eta$, then there is some $\lambda \in \mathcal{F}$ such that $\lambda \leq \eta$ and hence $Y . c \ell(\lambda) \leq Y . c \ell(\eta)$. So $1_{X} q Y . c \ell(\eta)$. So, in any case, $1_{X} q Y . c \ell(\eta)$ and, consequently, as $Y . c \ell(\eta)=Y . c \ell\left(\rho_{0}\right), 1_{X} q Y . c \ell\left(\rho_{0}\right)$. Thus $y_{\alpha}^{0} \in Y . \theta S . c \ell(X)$. So, $y_{\alpha}^{0} \in S(\Psi, x)$ for some $x \in X$. Consider any $V \in S O N_{x}$ and $\lambda \in \mathcal{F}$. Then $\lambda \vee\left\{y_{\alpha}^{0}\right\} \in \Delta$ and $y_{\alpha}^{0} q Y \backslash\left(\lambda \wedge\left\{y_{\alpha}^{0}\right\}\right)$. Thus $Y \backslash\left(\lambda \vee\left\{y_{\alpha}^{0}\right\}\right) \in \Delta$, which proves that $Y . c l\left(\lambda \vee\left\{y_{\alpha}^{0}\right\}\right)=\lambda \vee\left\{y_{\alpha}^{0}\right\}$. Now, since $Y . c \ell\left(\lambda \vee y_{\alpha}^{0}\right) q \Psi(X . c \ell(V))$, then $X . c \ell(V) q \lambda \vee y_{\alpha}^{0}$ which implies that $X . c \ell(V) q \lambda$. Thus, $x_{\alpha} \in \theta \operatorname{S.adh}(\mathcal{F})$. 


\section{References}

[1] M. E. Abd El-Monsef, I. M. Hanfy and S. N. El-Deeb, On fuzzy $\delta$-continuity and fuzzy $\delta$-closed graph, Mansoura Sci. Bull. 17 (1990), 11-25.

[2] K. K. Azad, Fuzzy grills and a characterization of fuzzy proximity, J. Math. Anal. Appl. 79(1981), 13-17.

[3] C. L. Chang, Fuzzy topological spaces, J. Math. Anal. Appl. 24(1968), 182-190.

[4] E. F. Collingwood and A. J. Lohwater, The theory of cluster sets, Cambridge University Press, Cambridge 1966.

[5] T. R. Hamlett, Cluster sets in general topology, J. London Math. Soc. 2(1975/1976), 192-198.

[6] J. E. Joseph, Multifunctions and cluster sets, Proc. Amer. Math. Soc. 74(1979), 329-337.

[7] A. Kandil, E. E. Kerre, A. A. Nouh and M. E. El-Shafei, Generalized mappings between fuzzy topological spaces, Mathematica Pannonica 31(1992), 59-71.

[8] E. E. Kerre, A. A. Nouh and A. Kandil, Generalized compactness in fuzzy topological spaces, Math. Vesnik 43(1991), 29-40.

[9] E. E. Kerre, A. A. Nouh and A. Kandil, Operations on the class of all fuzzy sets on a universe endowed with a fuzzy topoloty, J. Math. Anal. Appl. 180 (1993), 325-341.

[10] M. N. Mukherjee and S. Malakar, On almost continuous and weakly continuous fuzzy multifunctions, Fuzzy Sets and Systems 41 (1991), 113-125.

[11] A. A. Nouh, $\theta$-convergence theory of fuzzy filters, J. Fuzzy Math. 8 (2000), 893-908.

[12] Pu Pao-Ming and Liu Ying-Ming, Fuzzy topoloty I, J. Math. Anal. Appl. 76 (1980), 571-599.

[13] S. Sampath Kumar, Almost continuous and weakly continuous fuzzy set valued functions on bitopological spaces, Fuzzy Sets and System 112 (2000), 263-269.

[14] J. D. Weston, Some theorems on cluster sets, J. London Math. Soc. 33 (1958), 435-441.

Mathematics Department, Faculty of Science, Mansoura University, Mansoura 35516, Egypt. 